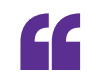

The LEVO-

CTS trial ... showed a

trend towards

lower 90-day

mortality with

levosimendan
$\Rightarrow$ SURGERY

\section{Neutral results for levosimendan in cardiac surgery}

Prophylactic use of levosimendan does not reduce the rate of death, renal replacement therapy, perioperative myocardial infarction, or use of a mechanical cardiac assist device in patients undergoing cardiac surgery, according to findings from the LEVO-CTS trial presented at ACC.17. "Despite neutral results on these primary outcomes," says Rajendra Mehta, lead investigator of the trial, "our data showed that the drug increased cardiac output and decreased low-cardiac-output syndrome, without an increase in adverse events."

Nevertheless, the investigators acknowledge that findings on these secondary end points should be considered exploratory.

According to Mehta, including use of a mechanical assist device as a primary end point might have contributed considerably to the neutral results, because physician decisions varied widely among centres. The LEVO-CTS trial, which included 882 patients with left ventricular ejection fraction $\leq 35 \%$ who were undergoing cardiac surgery, also showed a trend towards lower 90-day mortality with levosimendan than with placebo, although the trial was not powered to detect significant differences in mortality. This observation contrasts with results from the CHEETAH trial showing that low-dose levosimendan was not associated with lower 30-day mortality in patients after cardiac surgery compared with placebo. This trial was stopped early for futility. In view of the available evidence on the beneficial effects of levosimendan on cardiac output, low-cardiac-output syndrome, and absence of adverse events, Mehta believes this drug is a reasonable option in patients undergoing cardiac surgery in which increased cardiac output is the desired goal.

Irene Fernández-Ruiz

ORIGINAL ARTICLES Mehta, R. H. et al. Levosimendan in patients with left ventricular dysfunction undergoing cardiac surgery. N. Engl.J. Med. http://dx.doi.org/10.1056/ NEJMoa1616218 (2017) | Landoni, G. et al. Levosimendan for hemodynamic support after cardiac surgery. N. Engl. J. Med. http://dx.doi.org/10.1056/NEJMoa1616325 (2017) 